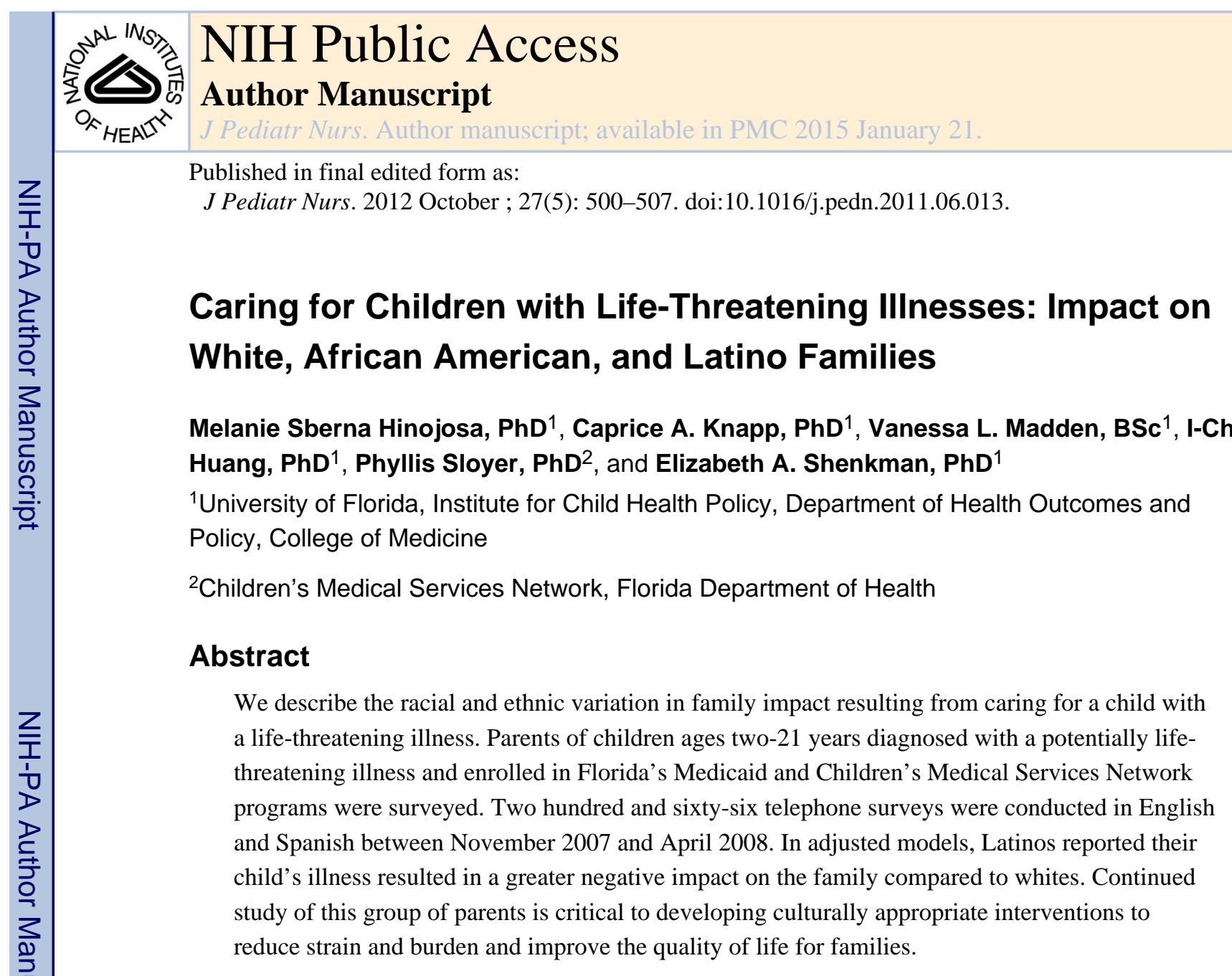

\title{
Keywords
}

children; life-threatening illnesses; race/ethnicity; family; caregiving

Children with special health care needs (CSHCN) make up approximately $14 \%$ of children and youth in the United States (CAHMI, 2010). There are currently 16.8 million unpaid caregivers providing care to these children, and a recent study reveals one-half of CSHCN were assisted by caregivers with at least one activity of daily living related to the child's medical conditions (Caregiving \& AARP, 2009). Most assistance is provided in dressing, bathing, and mobility, however, some caregivers are also being expected to provide more inhome intensive treatments, such as administering intravenous medications to their children (Wells et al., 2002). Family caregivers report spending an average of 30 hours per week caring for their child's illness, and almost $40 \%$ of caregivers surveyed report being in high burden situations based on level of care provided (Caregiving \& AARP, 2009). Family caregivers are impacted by higher levels of burden, uncertainty, greater depressive symptoms, poor quality of life, poor health, and increased mortality (Brehaut et al., 2009; Caregiving \& AARP, 2009; Hutchinson, Willard, Hardy, \& Bonner, 2009; Kuhlthau, Kahn, Hill, Gnanasekaran, \& Ettner, 2010; Vitaliano, Zhang, \& Scanlan, 2003). In this paper we

Please direct correspondence to: Melanie Sberna Hinojosa, $\mathrm{PhD}$, University of Florida, Institute for Child Health Policy, Department of Health Outcomes and Policy, College of Medicine, PO Box 100177, Gainesville, FL 32610-0177. Phone: 352-265-0111 x85736. Fax: 352-265-8047. mhinojosa@ichp.ufl.edu. 
explore the impact of caregiving for children with life-threatening illnesses, defined as illnesses that place the child at risk of dying before he/she reaches adulthood. Using the Impact on Family Scale, we examine the variation in family impact for white, African American, and Latino families who participate in Florida's public insurance program.

\section{Family Caregiving}

Family caregivers provide a distinct set of services for their children, which can cause stress and burden on the family. Because family caregivers are not trained medical professionals, they may often feel uncertain about their ability to manage the caregiving role at home (Berg, Anderson, Tichacek, Tomizh, \& Rachelefsky, 2007). This uncertainty can result in increased stress and anxiety within the family. Beyond the psychosocial difficulties that a child's illness brings to the family, the family can also suffer financially (Bumbalo, Ustinich, Ramcharran, \& Schwalberg, 2005). One or both parents might have to reduce work hours or give up employment if the demands of caring for his/her child outweigh the benefits of employment. Caregivers of children with greater functional limitations, caregivers with lower socioeconomic status, and non-native English speaking caregivers are most at risk for loss of employment or reduced work hours (Okumura, Van Cleave, Gnanasekaran, \& Houtrow, 2009; Yu \& K, 2009). Out-of-pocket expenses and cost sharing mechanisms can also result in added financial burden, even for insured families.

The impact on family caregivers is often greater for children with life-threatening illnesses due to the intensity of caregiving activities and the impact on other close family members and friends. Because no parent wants to be told that their child is at risk for dying, negative psychosocial impacts can be associated with the diagnosis of a life-threatening illness. Studies have shown that parents grieve for their child's loss of health as the child's illness progresses, although the intensity of the parent's grief varies (Armstrong-Dailey \& Zarbock, 2009; Miles \& Demi, 1983).

These difficulties may be exacerbated for racial and ethnic minority families and families from lower socioeconomic backgrounds. A disproportionate burden of special health care needs in the United States falls on racial and ethnic minority families, non-English speaking families, and families with lower socioeconomic status (Newacheck et al., 1998). These families also face multiple disparities in health, access to care, and use of primary care and specialty services (Flores \& Tomany-Korman, 2008; Porterfield \& CMcBride, 2007; Yu \& $\mathrm{K}, 2009)$. Lower levels of education and English proficiency are linked to lower health literacy levels (Sanders, Thompson, \& Wilkinson, 2007), which can create difficulties when caregivers need to administer complicated medications or follow strict care instructions.

Studies of caregivers of adults and elderly family members who have chronic and acute conditions (such as dementia, stroke, cancer, diabetes) have found racial and ethnic differences in caregiver stress, burden, injury, general health, and health-related quality of life (Glozman, 2004; Low, Payne, \& Roderick, 1999). White caregivers report more distress and depressive symptoms compared to African American caregivers (Janevic \& Connell, 2001), who are more likely to have favorable perceptions of their caregiving role (DilworthAnderson et al., 2005). Despite a more positive outlook on their caregiving role, African 
American caregivers have been found to have more health problems and higher mortality rates compared to whites (Dilworth-Anderson, Goodwin, \& Willians, 2004).

As a group, Latino caregivers report a greater sense of duty to provide care and spend more hours on informal care compared to whites and African Americans (Clark \& Huttlinger, 1998; Mausbach et al., 2004). Trends within pan-ethnic Latino groups in the US, originating from Mexico, Cuba, Puerto Rico, or other Latin American countries, are less transparent. These groups differ greatly from one another in their experiences with discrimination, migration, acculturation, and culture, all of which can affect health, health behaviors, and access to healthcare (Zsembik \& Fennell, 2005). Most caregiver studies to date treat Latinos as a monolithic group, despite their diverse histories, migration patterns, cultural beliefs, and experiences once in the United States (Eiraldi, 2004; Rothe, 2004). Overall, the racial and ethnic variations in family caregiving outcomes can be attributed to differences in cultural approaches to caregiving (Dilworth-Anderson et al., 2004), differences in caregiver family support networks (Hinojosa, Zsembik, \& Rittman, 2009), and greater intensity of caregiving duties due to excess burden of chronic disease in racial and ethnic minority populations.

While there is a plethora of literature on caregivers of adult family members, less is known about the variation in stress for white, African American, and Latino caregivers of children with life-threatening illnesses. The primary aim of this paper is to fill this gap in the literature through a descriptive analysis of racial and ethnic variation in family impact amongst caregivers of children with life-threatening illnesses enrolled in Florida's public insurance programs. Differences in caregiver impact within Latino pan-ethnic groups also remain largely unexplored; a secondary aim is to conduct a sub-analysis of these groups to determine if they differ in family impact.

\section{Method}

Study participants were parents of children aged two to 21 years of age. In order to be enrolled, the child had to have been diagnosed with a potentially life-threatening illness and enrolled in Florida's Medicaid and Children's Medical Services Network (CMSN), the State's Title V program for CSHCN. Child participants were considered as having a potentially life-threatening illness if he/she met the enrollment criteria for the State's Partners in Care: Together for Kids (PIC:TFK) pediatric palliative care program. However, the children in this study were not enrolled in PIC:TFK because it was not available statewide at the time the study was conducted. The reader is referred to Knapp et al. (2010b) for a list of diagnoses that are considered eligible for the PIC:TFK program. An introductory letter was sent to 936 parents meeting the criteria, and telephone surveys were conducted in English and Spanish between November 2007 and April 2008. Four hundred eighty-nine of the potential subjects had invalid contact information. Overall, 266 surveys were completed, resulting in a response rate of 54.4\%. The Institutional Review Boards at both the University of Florida and the Florida Department of Health approved this study. 


\section{Participants}

Two hundred and sixty-six parents of racial and ethnic minority children with potentially life-threatening illnesses were enrolled in our study. We selected only those caregivers who were Latino, white, or African American. We eliminated individuals who had missing data across other variables of interest. For example, young adults ages 19-21 did not answer the Pediatric Quality of Life (PedsQL) Measure, and thus were excluded from the sample. There were a few other missing cases $(n<4)$ across some of the demographic variables and they were also excluded from our analysis. 12 individuals ( $4.5 \%$ of the sample) were categorized as "other race" which consisted of Asian American/Pacific Islander, American Indian/Native American, and "other". Because there were too few of these individuals to make accurate comparisons across races, they were eliminated from the analysis. Our final sample size for analysis was 213 .

\section{Outcome Measure}

Impact of the children's illness on their families was the primary outcome of this study and the Impact on Family (IOF) scale was included in the telephone survey (Stein \& Reiessman, 1980). The IOF Scale was developed to measure the impact that a wide variety of children's illnesses may have on families. A validation study by Stein and Jessop was conducted in 2003 and as a result, the IOF scale was revised from a 27 -item scale to a 15 -item scale. Studies have used the IOF scale to measure family impact on specific diseases as well. One study (Kromer, Prihoda, Hidalgo, \& Wood, 2000) used the 27 item IOF scale to measure the family impact of 115 Hispanic parents of children with asthma. The study found that total IOF score was correlated with access to health care and parental knowledge. Another study of 115 Icelandic parents of chronically ill children used the 24-item IOF scale and found that greater IOF scores were associated with more severe illness, more hospitalizations, and longer hospitalizations (Gudmundsdottir, Elkit, \& Gudmundsdottir, 2006). Knapp et al. (2010b) found that among families whose children were enrolled in a pediatric palliative care program, the IOF scale predicted parental depressive symptomatology and showed variation in racially and ethnically diverse families, with African American parents' displaying less negative impact on the family.

We utilize the Impact on Family 15 item scale (Stein, 2003) that measures social and personal strain. The response categories for each item are "strongly disagree", "disagree", "agree", and "strongly agree". Items are scored from one to four, where a four indicates the greatest impact, and the total IOF score is calculated by summing the item scores. A score of zero indicates low impact on family and a score of 60 indicates high impact on family. Missing values were imputed when the number of missing items on the IOF was four or fewer. Items were imputed by replacing the missing values with respondents' average (mean) response across all other items. No parents had more than four missing items on the IOF. We calculated the Cronbach alpha for the IOF scale in our sample: alpha $=0.85$.

\section{Sociodemographic Characteristics}

To determine which factors affected the IOF score, several demographic questions were included on the survey. These questions asked about parent's age, parent's race/ethnicity, parental language spoken at home, parent's marital status, parent's educational attainment, 
parent's relationship to child (parent or non-parent), type of household (single or two parent), number of years providing care for the illness, and child's gender.

\section{Child Health Variables}

Health was measured by parental report of health status. Parents were asked to rate their child's current health status on a scale of 1 to 10 , with 1 indicating the worst health and 10 representing the best health. We also included a measure of the child's health-related quality of life. The PedsQL Generic Version 4.0, a 23-item scale that measures the child's physical, emotional, social and school functioning, was included in the survey for children ages two to 18. The PedsQL response categories are "almost always", "often", "sometimes", "almost never", and "never" had a problem in the past month with each functioning item. Items are scored from one to five and the total PedsQL score is calculated by summing the item scores and linearly transforming them to a zero to 100 point score. Higher scores indicate better health-related quality of life.

\section{Analytic Strategy}

First, we conducted bivariate comparisons of the demographic characteristics by race and ethnicity using the appropriate method for the variable types (Chi Square, Fisher's Exact, or one-way analysis of variance). Next, we conducted a series of unadjusted bivariate logistic regression analyses of the $15 \mathrm{IOF}$ items to see how Latino and African American parents differ from whites ( $1=$ agree, $0=$ disagree). Finally, we used the IOF scale as an outcome variable in an ordinary least squares regression analysis, adjusting for race, child health, and demographic characteristics. Three models were tested. The first model controls for race and ethnicity, the second model adds child health variables, and the third model adds demographic characteristics. We established an a priori significance level of alpha=.05 or less.

\section{Results \\ Demographics}

Non-Hispanic whites made up $44.1 \%$ of the sample (see Table 1), non-Hispanic African Americans made up $25.4 \%$ of the sample, and Latinos (any origin) made up 30.5\% of the sample. Of those parents who indicated Latino ethnicity, 35.4\% were Puerto Rican, 24.6\% were Mexican American/Chicano, 15.4\% were Cuban, and 24.6\% identified as other Latino (including South American, Central American, and Other Hispanic).

Tables 2 and 3 display the results of Chi Square and/or Fisher's exact tests for the racial and ethnic variation in demographic and child health. The average child age was 10.5 years and did not differ significantly by race/ethnicity. The average age of the caregivers was 42.4 , with white caregivers averaging 45.2 years, African American caregivers 41.9 years, and Latino caregivers 38.9 years. A primary language other than English was spoken in $41.5 \%$ of Latino households compared to $2 \%$ or less in white and African American households. Latinos had the highest percentage of those married (69.2\%) followed by whites (50\%), and African Americans (25.9\%). Almost all caregivers in Latino households were the parents of the child they were caring for (96.9\%) compared to $81.5 \%$ of African American and $67 \%$ of 
white caregivers. Latinos had the highest percentage of two parent households at $67.7 \%$, compared to $55.3 \%$ of whites and $31.5 \%$ of blacks. Educational level also varied, with $30.8 \%$ of Latinos possessing less than a high school diploma followed by $24.1 \%$ of blacks, and $13.8 \%$ of whites.

Parents' ratings of child's health did not vary by race/ethnicity; on a scale from 1-10, the average rating was 7.4 for African Americans and 7.3 for Latinos and whites. Results of PedsQL ratings, measured on a scale of 0 to 100 with 100 representing the best health, also showed no significant racial or ethnic differences, with an average score of 57.46.

\section{Impact on Families By Race/Ethnicity}

There were racial and ethnic differences across 8 of the 15 items on the IOF scale (see Table 4). Stronger agreement with the items represents a negative impact on the family. Latinos were 2-3 times more likely to indicate stronger agreement for 5 items (Items 1, 2, 7, 12, \& 13) compared to whites: inability to travel $(\mathrm{OR}=2.05)$, special treatment by neighbors $(\mathrm{OR}=2.62)$, wondering about special treatment $(\mathrm{OR}=2.78)$, living life day to day $(\mathrm{OR}=2.82)$, and no one to understand the burden of illness $(\mathrm{OR}=2.49)$. African American parents indicated greater agreement with the statement (Item 8) that they thought about not having more children because of the illness ( $\mathrm{OR}=2.27)$. African American parents were also less likely to agree with (items $10 \& 11$ ) the statements that they had to give things up because of their child's illness $(\mathrm{OR}=0.35)$ and that fatigue is a problem for them because of the illness $(\mathrm{OR}=0.47)$.

Next, we focused on all Latinos an d partitioned them into pan-ethnic groups for comparison. Mexican Americans, Puerto Ricans, Cuban Americans and other Latino (including Central and South American groups) were compared across the 15 items in the IOF scale (table not shown). We obtained log odds of agreement with IOF statements using bivariate logistic regression analysis. We found no differences among pan-ethnic groups across any of the individual IOF items.

Table 4 displays the results of the ordinary least squares (OLS) regression analyses with the IOF summary score as the dependent variable. A normal probability plot was created to confirm that the IOF scale has a normal distribution, and thus is appropriate for OLS regression analysis. We tested three models to test the impact of the predictor variables. Model 1 indicates that Latinos report significantly higher levels of impact (more negative outcome) on the family. Latinos have a score 2.87 points higher ( $\mathrm{p}<.05)$ than white caregivers. In Model 1, the adjusted R Square statistic indicates that in the model with race/ ethnicity alone, we are only accounting for $2.54 \%$ of the variance in the IOF scale. In Model 2, Latinos continue to have a significantly higher impact on family score compared to whites $(b=3.11, p<.01)$. Additionally, general health rating and HRQOL are associated with the impact on family scale. Parents that report their child is in better health indicate significantly lower impact on the family $(b=-0.508, \mathrm{p}<.05)$. Better assessments of HRQOL corresponded to significantly lower impact on the family $(b=-.0195, \mathrm{p}<.01)$. Adding the health characteristics improves the amount of explained variance in IOF scale with an adjusted $R^{2}$ of $27.04 \%$. 
Model 3 adds the sociodemographic characteristics of families and caregivers. Latino caregivers in the full model still report greater impact on the family compared to whites, though the effect is diminished slightly $(b=2.78, \mathrm{p}<.01)$. Greater severity of illness continues to be associated with greater impact on family $(b=3.37, \mathrm{p}<.01)$. Better health ratings ( $b=-$. $72, \mathrm{p}<.01)$ and HRQOL continue to be negatively associated with impact on family ( $b=-.19$, $\mathrm{p}<.01)$. The demographic characteristics do not appear to add any additional explanatory power to the model; in fact, the amount of explained variance drops slightly $\left(R^{2}=.2682\right)$ when they are added.

\section{Discussion}

We have found significant variation in family impact for a sample of racially and ethnically diverse family caregivers whose children have life-threatening illnesses. Compared to whites, we found Latino parents to have higher scores on the IOF scale, even after controlling for child's health and sociodemographic characteristics. This finding that lifethreatening illnesses in Latino children correspond to greater stress and burden for Latino families than for white families is consistent with the literature on Latino caregivers of adults with chronic or disabling illnesses. Latino family caregivers are more likely to experience depression compared to whites and African Americans (Janevic \& Connell, 2001). At the same time, Latinos feel a greater sense of duty toward caregiving (Clark \& Huttlinger, 1998; Phillips, De Ardon, Komnenich, Killeen, \& Rusinak, 2000) and indicate greater reluctance to institutionalization (Mausbach, et al., 2004).

A unique aspect of this paper is that we included a sizeable proportion of African Americans and Latinos, as well as the often overlooked Latino pan-ethnic Mexican American, Cuban American, Puerto Rican and other Latino groups. To date, very few studies have addressed the potential differences in family caregiving experiences amongst pan-ethnic subgroups. Although the sample size was very small for the pan-ethnic groups, their inclusion is important because they can differ in family size, cultural conventions, acculturation, and attitudes toward medical care (Eiraldi, 2004; Rothe, 2004). Although we did not find any significant differences between the pan-ethnic groups during this study, this may have been due to limited sample sizes of each of the subgroups. Future research should continue to investigate the differences in these subgroups with larger sample sizes.

Compared with other groups, Latinos as a group were also more likely to endorse statements about inability to travel and not being able to plan for the future because of their child's illness. This finding could be especially prominent for Latino families because of an increased likelihood of family living at greater distances and an increased cultural importance of travelling to see extended family. Latino parents also indicated they felt neighbors and friends treated their child specially and revealed their own uncertainty about whether to treat the children as special compared to healthy children. Finally, Latino parents were two and a half times more likely to state that no one understood the burden of care they carry. It is possible this could be due to hesitancy of Latino caregivers to join existing support groups if they are not in their native language or include parents from similar backgrounds. This would suggest a need for health care organizations or community advocates to develop and implement Latino-specific support groups for parents of children 
with life-threatening illnesses. Clinicians could play a more active role in addressing Latino parental impact by providing information about available psychosocial services and tips on how to lessen negative impacts. For example, a nurse care coordinator or patient navigator could educate parents on how to plan for travelling with their ill child, manage insurance, care for their child at home, and recognize when their own feelings of burden may need to be treated by professionals.

African Americans did not significantly differ from whites in regard to impact on the family in the adjusted models. However, in the unadjusted models African American family caregivers of children with life threatening illnesses differed from whites on feelings of fatigue and giving up things due to child's illness (Items 10 and 11). African Americans were less likely to feel fatigue and less likely to report giving up things due to the child's illness than whites. This is consistent with the literature on adult caregiving, which reports African Americans as more likely to cite cultural reasons for wanting to provide care, as well as lower feelings of burden and depression compared to white caregivers (DilworthAnderson et al., 2005; Janevic \& Connell, 2001; Roff et al., 2004).

There are several limitations to this study worth noting. First, this study was conducted with a sample of parents whose children receive health care through the Medicaid program in the State of Florida. This sample may limit the generalizability of our findings, as no assumption can be made regarding the experiences of caregivers of children with lifethreatening illnesses who receive health care through private insurance plans. Moreover, Florida has a large racial and ethnic minority population, many of whom are Latino. Our results may have been different for parents who reside in less racially and ethnically diverse states. Third, the majority of respondents in our survey were mothers. We cannot speculate on the congruence or incongruence of reported burden between mothers and fathers. Future research should address this important area. Fourth, we used the PedsQL as a measure of health status instead of using the children's individual diagnoses. Many of the children in PIC:TFK have more than one life threatening illness or have a combination of diagnoses that result in threatening status. Therefore, if we had accounted for the child's specific diagnosis, we would not have been able to interpret the results in a meaningful way. Fifth, when a child has a life threatening illness, families can experience stress and burden not only related to the illness, but also from changes in family dynamics and health system factors. We plan to conduct future research on health system factors, including involvement in making decisions, care coordination, and the level of trust in the child's physicians, and potential interventions that may be used to ease families' burden. Finally, though our response rate of $54 \%$ was consistent with our prior telephone survey response rates with this population, we did not compare the characteristics of responders and non-responders.

Despite these limitations, this study is novel in that we explore important variations in racial and ethnically diverse families with children with life-threatening illnesses. Through this study we have begun to understand the burden that racial and ethnic minority parents face when caring for a child with a life-threatening illness. Understanding that there are racial and ethnic differences in the levels of burden can lead to the development of tailored, culturally appropriate interventions to help improve outcomes for families and children. 


\section{References}

Armstrong-Dailey, A.; Zarbock, SF. Hospice care for children. 3rd ed.. New York: Oxford University Press; 2009.

Berg J, Anderson NLR, Tichacek MJ, Tomizh AC, Rachelefsky G. One gets so afraid: latino families and asthma management--An exploratory study. Journal of Pediatric Health Care. 2007; 21:361371. [PubMed: 17980802]

Brehaut JC, Kohen DE, Garner RE, Miller AR, Lach LM, Klassen AF, Rosenbaum PL. Health among caregivers of children with health problems: Findings from a Canadian population-based Study. American Journal of Public Health. 2009; 99(7)

Bumbalo J, Ustinich L, Ramcharran D, Schwalberg R. Economic impact on families caring for children with special health care needs in New Hampshire: The effect of socioeconomic and healthrelated factors. Maternal and Child Health Journal. 2005; 9s(2)

CAHMI, CaAHMI. 2005/2006 National survey of children with special health care needs. 2010 from Data Resource Center for Child and Adolescent Health Website www.cshendata.org.

Caregiving, NAf; AARP. Caregivers of children: A focused look at those caring for a child with special needs under the age of 18. Foundation, M., editor. Bethesda, MD: 2009.

Clark M, Huttlinger K. Elder care among Mexican American families. Clin Nurs Res. 1998; 7(1):6481. [PubMed: 9526315]

Dilworth-Anderson P, Brummett BH, Godwin P, Williams SW, Williams RB, Siegler IC. Effect of race on cultural justifications for caregiving. Journals of Gerontology: Social Sciences. 2005; 60(5):s257-s262.

Dilworth-Anderson P, Goodwin PY, Willians SW. Can culture help explain the physical health effects of caregiving over time among African American caregivers? Journals of Gerontology: Social Sciences. 2004; 59B(3):S138-s145.

Eiraldi RB. Treatment of ADHD in Latino populations: Developing cultural competency for multiple populations is the major challenge. Behavioral Health Management. 2004 Jul-Aug;

Flores G, Tomany-Korman SC. Raical and ethnic disparities in medical and dental health, access to care, and use of services in US children. Pediatrics. 2008; 121:e286-e298. [PubMed: 18195000]

Glozman JM. Quality of life of caregivers. Neuropsychology Review. 2004; 14(4):183-196. [PubMed: 15796115]

Gudmundsdottir HS, Elkit A, Gudmundsdottir DB. PTSD and psychological distress in Icelandic parents of chronically ill children: Does social support have an effect on parental distress? Scandinavian Journal of Psychology. 2006; 47:303-312. [PubMed: 16869863]

Hinojosa M, Zsembik B, Rittman M. Patterns of informal care among Puerto Rican, African American, and White stroke survivors. Ethnicity and Health. 2009; 14(6):591-606. [PubMed: 19670066]

Hutchinson KC, Willard VW, Hardy KK, Bonner MJ. Adjustment of caregivers of pediatric patients with brain tumors: A cross-sectional analysis. Psycho-Oncology. 2009; 18:515-523. [PubMed: 18756585]

Janevic MR, Connell CM. Racial, ethnic, and cultural differences in the dementia caregiving experience: Recent findings. Gerontologist. 2001; 41(3):334-347. [PubMed: 11405431]

Knapp C, Madden V, Curtis C, Sloyer P, Huang IC, Thompson LA, Shenkman EA. Partners in care: together for kids: Florida's model of pediatric palliative care. Journal of Palliative Medicine. 2010; 11(9):1212. [PubMed: 19021484]

Knapp C, Madden V, Curtis C, Sloyer P, Shenkman E. Family support in pediatric palliative care: How are families impacted by their children's illnesses? Journal of Palliative Medicine. 2010; 13(4)

Kromer ME, Prihoda TJ, Hidalgo HA, Wood PR. Assessing quality of life in Mexican-American children with asthma: Impact-on-family and functional status. Journal of Pediatric Psychology. 2000; 25:415-426. [PubMed: 10980046]

Kuhlthau K, Kahn R, Hill KS, Gnanasekaran S, Ettner SL. The well-being of parental caregivers of children with activity limitations. Maternal Child Health. 2010; 14:155-163. 
Low JT, Payne S, Roderick P. The impact of stroke on informal carers: a literature review. Soc Sci Med. 1999; 49(6):711-725. [PubMed: 10459884]

Mausbach BT, Coon DW, Depp C, Rabinowitz YG, Wilson-Arias E, Kraemer HC, GallagherThompson D. Ethnicity and time to institutionalization of dementia patients: A comparison of Latina and Caucasian female family caregivers. J Am Geriatr Soc. 2004; 52(7):1077-1084. [PubMed: 15209644]

Miles MS, Demi AS. Toward the development of a theory of bereavement guilt: Sources of guilt in bereaved parents. Omega. 1983; 14:299-314.

Newacheck PW, Strickland B, Shonkoff JP, Perrin JM, McPherson M, McManus M, Arango P. An epidemiologic profile of children with special health care needs. Pediatrics. 1998; 102:117-123. [PubMed: 9651423]

Okumura MJ, Van Cleave J, Gnanasekaran S, Houtrow A. Understanding factors associated with work loss for families caring for CSHCN. Pediatrics. 2009; 124:s392-s398. [PubMed: 19948604]

Phillips LR, De Ardon ET, Komnenich P, Killeen M, Rusinak R. The Mexican American caregiving experience. Hispanic Journal of Behavioral Science. 2000; 22(3):296-313.

Porterfield SL, CMcBride TD. The effect of poverty and caregiver education on perceived need and access to health services among children with special health care needs. American Journal of Public Health. 2007; 97(2)

Roff LL, Burgio LD, Gitlin L, Nichols L, Chaplin W, Hardin JM. Positive aspects of Alzheimer's caregiving: The role of race. Journals of Gerontology: Social Sciences. 2004; 59(3):185-190.

Rothe EM. Hispanic adolescents and their families: Sociocultural factors and treatment considerations. Adolescent Psychiatry. 2004; 28:251-278.

Sanders LM, Thompson VT, Wilkinson JD. Caregiver health literacy and the use of child health services. Pediatrics. 2007; 119(1)

Stein REK. The impact on family scale revisited: Further psychometric data. Journal of Developmental and Behavioral Pediatrics. 2003; 24(1):9. [PubMed: 12584480]

Stein REK, Reiessman CK. The development of an impact on family scale: Preliminary findings. Medical Care. 1980; 18(4):465-472. [PubMed: 7401703]

Vitaliano PP, Zhang J, Scanlan JM. Is caregiving hazardous to one's physical health?: A meta-analysis. Psychological Bulletin. 2003; 129(6):946-972. [PubMed: 14599289]

Wells DK, James K, Stewart JL, Moore IM, Kelly KP, Moore B, Speckhart B. The care of my child with cancer: A new instrument to measure caregiving demand in parents of children with cancer. Journal of Pediatric Nursing. 2002; 17(3)

Yu SM, K SG. Household language use and health care access, unmet need, and family impact among CSHCN. Pediatrics. 2009; 124:s414-s419. [PubMed: 19948607]

Zsembik B, Fennell D. Ethnic variation in health and the determinants of health among Latinos. Social Science and Medicine. 2005; 61:53-63. [PubMed: 15847961]

J Pediatr Nurs. Author manuscript; available in PMC 2015 January 21. 
Table 1

Race and Ethnicity of Sample

\begin{tabular}{llll}
\hline & & $\mathbf{n}$ & $\%$ \\
\hline Caregiver Race/Ethnicity & Latino & 65 & $30.5 \%$ \\
& White & 94 & $44.1 \%$ \\
& Black & 54 & $25.4 \%$ \\
& Total & 213 & $100.0 \%$ \\
Caregiver Hispanic Ethnicity & Latino & 65 & $30.5 \%$ \\
& Non-Latino & 148 & $69.5 \%$ \\
& Total & 213 & $100.0 \%$ \\
& Mexican American & 16 & $24.6 \%$ \\
& Puerto Rican & 23 & $35.4 \%$ \\
& Cuban American & 10 & $15.4 \%$ \\
& South American, Central American \& Other Hispanic/Latino & 16 & $24.6 \%$ \\
& Total & 65 & $100.0 \%$ \\
\hline
\end{tabular}




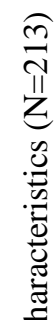

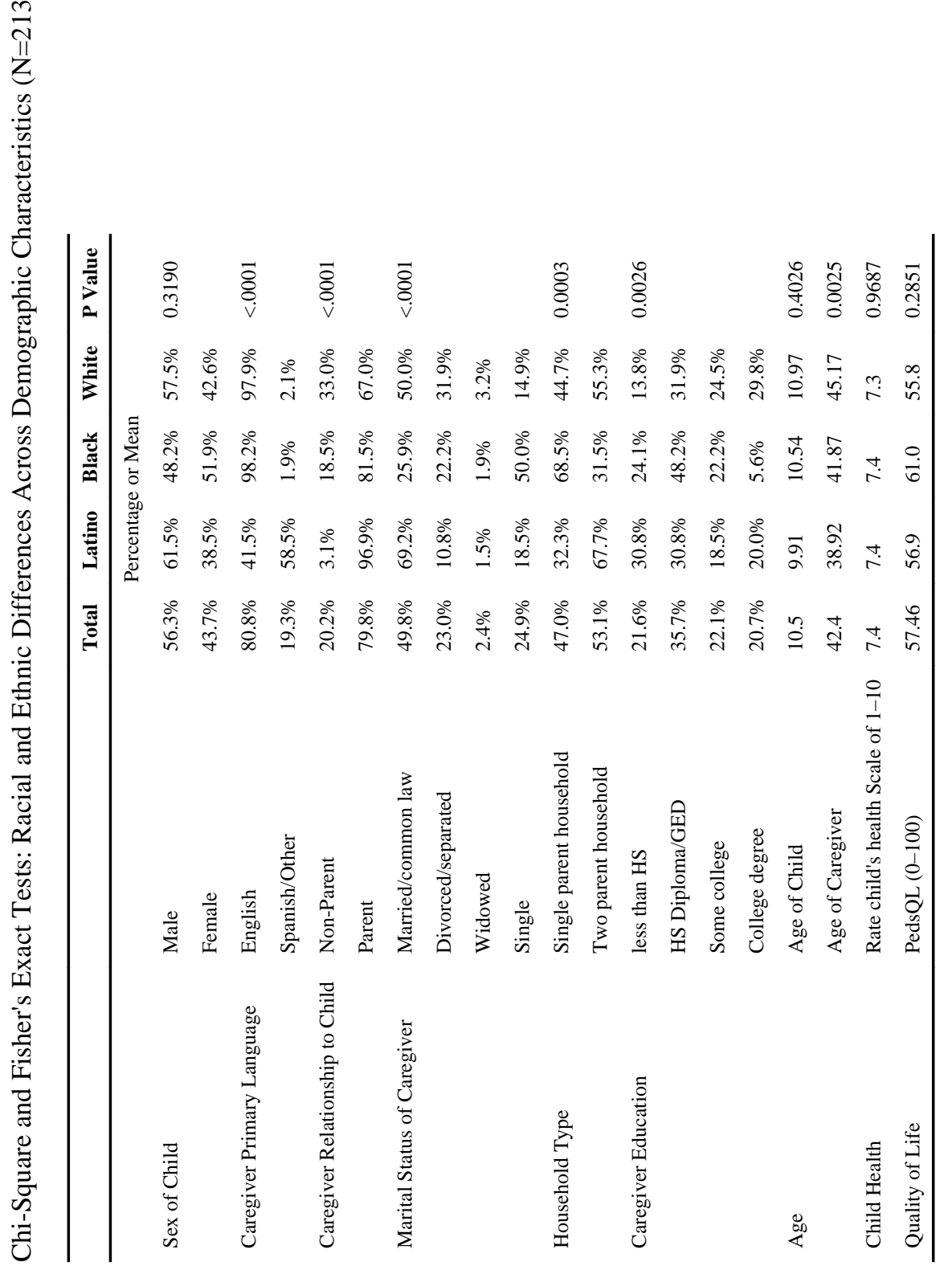


Table 3

Log Odds of Caregiver Reporting They Strongly Agree or Agree with the Statement

\begin{tabular}{|c|c|c|c|c|}
\hline & Latino & 95\% CI & Af. Amer. & $95 \% \mathrm{CI}$ \\
\hline Because of the illness we are not able to travel out of the city & 2.05 & 1.06 to 3.97 & 1.37 & 0.67 to 2.77 \\
\hline People in the neighborhood treat us special because of my child's illness & 2.62 & 1.24 to 5.52 & 2.05 & 0.93 to 4.54 \\
\hline We have little desire to go out because of my child's illness & 1.49 & 0.76 to 2.92 & 0.64 & 0.29 to 1.41 \\
\hline It is hard to find a reliable person to take care of my child & 1.39 & 0.70 to 2.75 & 0.51 & 0.26 to 1.00 \\
\hline $\begin{array}{l}\text { Sometimes we have to change plans about going out at the last minute because of my } \\
\text { child }\end{array}$ & 1.41 & 0.74 to 2.69 & 0.84 & 0.43 to 1.68 \\
\hline We see family and friends less because of the illness & 1.44 & 0.74 to 2.80 & 0.57 & 0.26 to 1.27 \\
\hline Sometimes I wonder if my child should be treated 'special' or the same as a normal child & 2.78 & 1.43 to 5.42 & 1.30 & 0.66 to 2.60 \\
\hline I think about not having more children because of the illness & 1.20 & 0.59 to 2.45 & 2.27 & 1.09 to 4.71 \\
\hline I don't have much time left over for other family members after caring for my child & 1.21 & 0.64 to 2.29 & 0.59 & 0.29 to 1.21 \\
\hline Our family gives up things because of my child's illness & 1.19 & 0.63 to 2.25 & 0.35 & 0.17 to 0.71 \\
\hline Fatigue is a problem for me because of my child's illness & 0.80 & 0.42 to 1.52 & 0.47 & 0.24 to 0.93 \\
\hline I live from day to day and don't plan for the future & 2.82 & 1.46 to 5.42 & 1.27 & 0.63 to 2.55 \\
\hline Nobody understands the burden I carry & 2.49 & 1.30 to 4.78 & 1.67 & 0.84 to 3.31 \\
\hline Traveling to the hospital is a strain on me & 1.04 & 0.54 to 1.99 & 0.78 & 0.39 to 1.57 \\
\hline Sometimes I feel like we live on a rollercoaster & 1.35 & 0.68 to 2.70 & 0.65 & 0.33 to 1.30 \\
\hline
\end{tabular}

Note. Numbers in the body of the table represent the log odds of "strongly agreeing" or "agreeing" with the statements at the left compared to disagreeing or strongly disagreeing. 


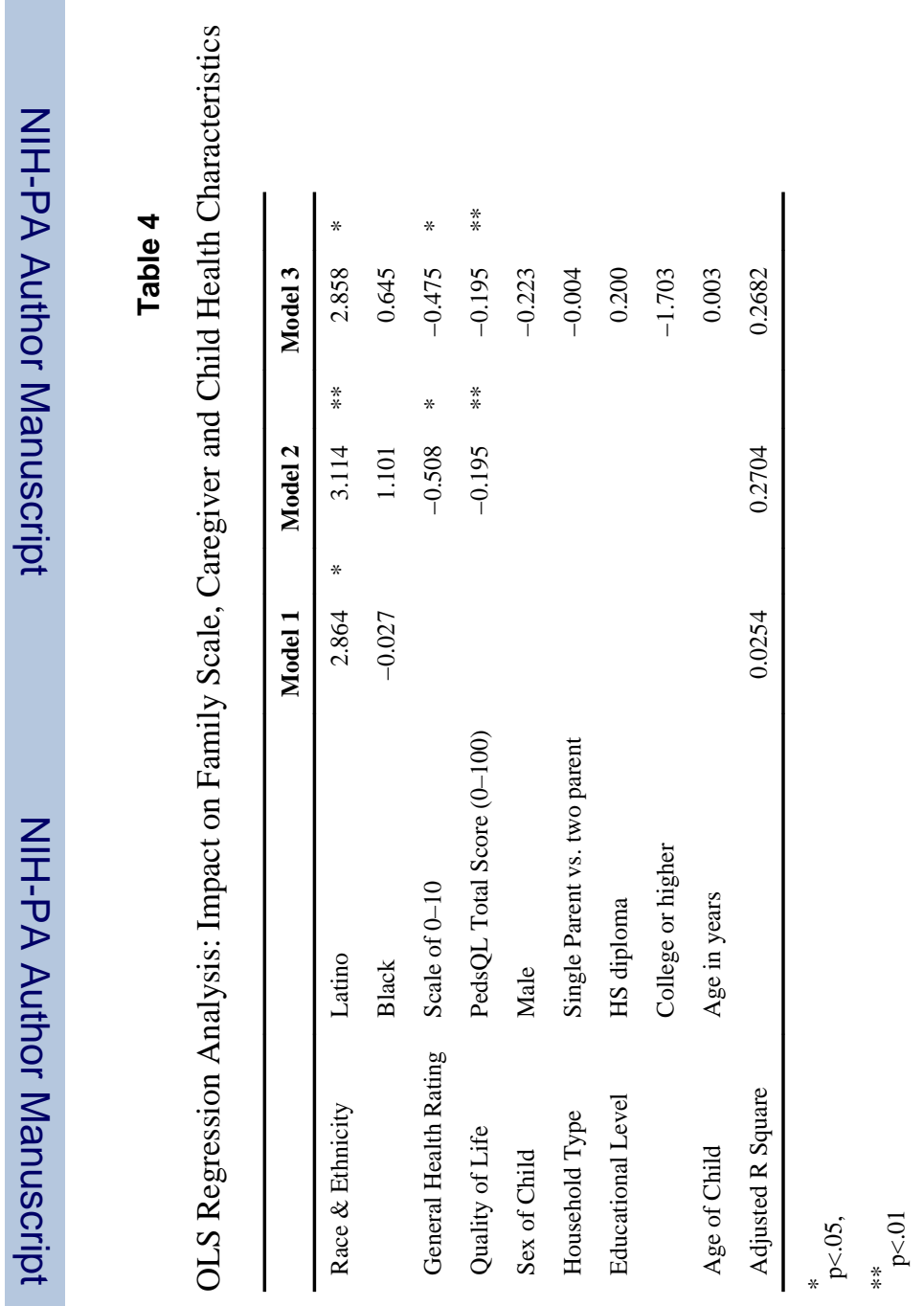

J Pediatr Nurs. Author manuscript; available in PMC 2015 January 21. 